Original Article

\title{
Taping of the elbow extensor muscle in chronic stroke patients: comparison between before and after three-dimensional motion analysis
}

\author{
Dong-Hun LeE ${ }^{1)}$, Won-Jin Kim ${ }^{2)}$, JAe-Seop $\mathrm{OH}^{3)}$, Moonyoung $\mathrm{CHANG}^{4)^{*}}$ \\ 1) Department of Occupational Therapy, Medwill Hospital, Republic of Korea \\ 2) Department of Occupational Therapy, College of Natural Sciences, Kosin University, Republic of \\ Korea \\ 3) Department of Physical Therapy, College of Biomedical Science and Engineering, Inje University, \\ Republic of Korea \\ 4) Department of Occupational Therapy, College of Biomedical Science and Engineering, Inje \\ University: 197 Inje Street, Gimhae, Gyeongsangnam-do 621-749, Republic of Korea
}

\begin{abstract}
Purpose] This study aimed to observe the effect of kinesio taping on the quality of movement of each arm during a reaching task in patients with right-sided hemiparetic stroke. [Subjects and Methods] Sixteen righthanded participants who had had a right-sided hemiparetic stroke were requested to perform a reaching task with each arm, with and without kinesio taping. A three-dimensional motion analysis system was used to measure peak angular velocity, time to reach peak angular velocity, and movement units during elbow motion. [Results] In the right arm, movements during the reaching task with kinesio taping were faster, smoother, and more efficient than those without kinesio taping. The peak angular velocity increased, and the time to reach peak angular velocity decreased. Movement units decreased significantly. However, in the untaped arm, the movement was slower, rougher, and less efficient. [Conclusion] Kinesio taping provided a positive effect on the reaching movement of the taped arm of right-handed persons who had had a right-sided hemiparetic stroke.

Key words: Kinesio taping, Reaching, Stroke
\end{abstract}

(This article was submitted Feb. 19, 2015, and was accepted Mar. 24, 2015)

\section{INTRODUCTION}

Improvement of motor functions after stroke is important for patients ${ }^{1)}$. The ability to live independently after a stroke depends on the recovery of motor functions, particularly those of the upper limb ${ }^{2}$.

Kinesio taping is widely used in the field of rehabilitation both for treatment and prevention of sports-related injuries ${ }^{3)}$. It improves muscle strength through excitation of gamma motor nerves in the skeletal muscle, as the taped part raises the tension of the fiber ${ }^{4,5)}$. Kim et al. $\left.{ }^{6}\right)$ also noted that home application of kinesio taping to hemiplegic stroke patients resulted in significant improvements in activities of daily living, joint range of motion (ROM), and hand motions.

Controlled upper extremity movements are critical to performing various activities of daily living ${ }^{7}$. Studies to evaluate the effectiveness of upper limb rehabilitation in stroke patients are ongoing; these studies are evaluating the

*Corresponding author. Moonyoung Chang (E-mail: myot@ inje.ac.kr)

(C2015 The Society of Physical Therapy Science. Published by IPEC Inc. This is an open-access article distributed under the terms of the Creative Commons Attribution Non-Commercial No Derivatives (by-ncnd) License $<$ http://creativecommons.org/licenses/by-nc-nd/3.0/> . behavior of the extension $\operatorname{arm}^{8}$. Upper limb rehabilitation provides patients with the opportunity to practice and improve their ability to adjust their reach extent for different distances, and to produce peak and mean velocities appropriate for the distance ${ }^{9)}$.

Behavior analysis studies of task performance in arm reaching among chronic stroke patients have been performed, but no studies have been conducted on taping methods used as interventions. Therefore, this study aimed to investigate differences in peak angular velocity, time to reach peak angular velocity, and total movement units of arm reaching after applying taping to the elbow joint on the affected side of chronic stroke patients.

\section{SUBJECTS AND METHODS}

Sixteen survivors of left unilateral cerebral vascular accidents were recruited from Medwill Hospital in Busan, Republic of Korea. All the participants, which included 14 males (mean \pm SD age, $49.5 \pm 15.62$ years) and 2 females (58.5 \pm 7.77 years), demonstrated right-sided hemiparetic stroke and left-hand dominance. The stroke events occurred 6 months or more before the study (mean \pm SD poststroke period, $17.43 \pm 11.2$ months).

The recruitment criteria for participants were as follows: (1) right-sided hemiparetic stroke; (2) at least a 6-month 
poststroke period; (3) ability to reach the target $(400 \mathrm{~mm}$ forward, $700 \mathrm{~mm}$ high); absence of (4) elbow joint contracture, (5) unilateral neglect, (6) visual deficit, and (7) apraxia; and (8) ability to maintain a seated position in a chair. The study methodology was described to all the participants, who agreed to join this study voluntarily. This study was approved by the Inje University Ethics Committee for Human Investigations prior to their participation. Data were collected by using a three-dimensional motion analysis device (CMS-70P, zebris Medizintechnik GmbH, Isny, Germany), a three-dimensional (3-D) motion analysis system. The system includes a laptop computer, active markers $(10 \mathrm{~mm}$ in diameter), a basic unit, a cable adapter, and a measuring sensor (MA-70P). The measuring sensor received the ultrasound signals released by active markers, and then the motion of the active markers in 3-D space was processed by using the computer. Data were sampled through WinData 2.19 (zebris Medizintechnik GmbH, Isny, Germany).

Taping was performed by an occupational therapist that was certified to perform taping. The subjects were taped in accordance with Kenzo Kase's Kinesiotaping Manual. Taping was applied to the elbow with the subject in a sitting position. A Y strip of tape was placed at the olecranon process of the elbow, stretched approximately to $120 \%$ of its maximum length, and attached just infraglenoid tubercle, posterior to the humerus above the spiral groove.

The task involved reaching to touch the top of a target while sitting in a seated position. The participants performed the reaching task with each arm under two conditions, namely with and without taping. The task was repeated five times in each condition. Each participant was seated in a standard chair $430 \mathrm{~mm}$ from the floor, with a seat depth of $410 \mathrm{~mm}$. The target was a cup (diameter, $62 \mathrm{~mm}$; height, $110 \mathrm{~mm}$ ) placed on a table (height: $740 \mathrm{~mm}$ ) in front of the participant. The subjects rested the arm to be used to perform movements on the table and held the cup in their hand; the other arm was placed on the knee. The kinematics of reaching to targets placed within and beyond the length of the arm were analyzed in the hemiparetic participants. Targets were placed sagittally in front of the midline of the body. Two targets (targets A and B) were within reaching distance, defined as the length of the stretched arm from the axilla to the wrist crease. The distance between the sternum and target A was $150 \mathrm{~mm}$. Target B was the distance to the point $70 \%$ of the arm length.

Compensatory movements of the trunk were restrained by securing the trunk of the participants to the backrest of the chair by using a 3-in-wide strap that ran across the chest. Three active markers were attached to the assigned arm as follows: on the styloid process of the radius, on the lateral epicondyle of the humerus, and on the midpoint of the humerus parallel to a virtual line between the second and third markers. Prior to performing the actual task to be recorded, all of the participants practiced to make sure they understood the instructions. When the researcher said "begin", the participant made a reaching movement from target A to target B. The participants were instructed to move at their fastest speed. Before every trial, each participant was positioned as described earlier. The task without taping was also performed.
Table 1. Within-subject comparison between untapped and taped arms in terms of elbow movement parameters during the reaching task $(\mathrm{n}=16)$

\begin{tabular}{lcc}
\hline & Untaped & Taped \\
\hline Peak velocity $(\% / \mathrm{s})$ & $248.4 \pm 107.3$ & $276.6 \pm 118.3^{*}$ \\
Time to peak velocity (ms) & $415.4 \pm 157.3$ & $346.7 \pm 144.5^{*}$ \\
Total movement units (units) & $10.7 \pm 4.9$ & $8.9 \pm 4.3^{*}$ \\
\hline
\end{tabular}

* Significance, $\mathrm{p}<0.05$

The dependent variables included the peak angular velocity, time to reach peak angular velocity, and movement units. The peak angular velocity indicated how fast elbow motion was during the reaching task. Time to reach peak angular velocity indicated the smoothness and efficiency of the motion. One movement unit was defined as the acceleration associated with deceleration in succession. An increase in movement units indicates that the participants made more corrections than before; therefore, the movement lacked smoothness and was less efficient.

The raw data were analyzed by using 3DAwin 1.02 . The start point of the reaching task was when the velocity of the first active marker increased from zero. The end point was when the velocity of the first active marker decreased to zero. The kinematic data were statistically analyzed by using the Statistical Package for the Social Sciences (SPSS) version 12.0 for Windows. A repeated-measures design was used to test within-subject statistically significant differences followed by repeated-measures analysis of variance. The alpha level was set at 0.05 for all the statistical analyses.

\section{RESULTS}

The means and standard deviations for each dependent variable are provided in Table 1. Taping increased the peak angular velocity, and decreased time to reach peak angular velocity and movement units in the taped arm. In the untaped arm, however, the peak angular velocity decreased, and the time to reach peak angular velocity and movement units increased. Significant differences were observed between the two conditions for all dependent variables.

\section{DISCUSSION}

This study investigated the effects of kinesio taping on elbow extensor muscles and compared them between the taped and untaped arms during reaching in right-sided hemiparetic stroke patients. The results showed that taping made elbow extensor function faster and more efficient in the taped arms but slower and less efficient in the untaped arms. This result supports our primary hypothesis that taping facilitated taped arm movement but not untaped arm movement.

In this study, taping was shown to have positive effects on right upper extremity movement in the right-handed patients with right-sided hemiparetic stroke. This result was similar to the findings in previous studies with healthy people, patients with cerebral palsy, and those with stroke ${ }^{10-12}$. Therefore, in patients with right-sided hemiparetic stroke, taping could facilitate right upper extremity movement. 
The elbow extensor offers static stability of the elbow joint. The extensor muscle group also functions to generate large and dynamic extensor muscle torque through a high rate of afferent or efferent activation. A general hierarchical recruitment pattern exists in elbow joint extensor muscle. The law of parsimony stipulates the mechanisms by which elbow joint extensor muscles are activated. In this case, the law of parsimony suggests the tendency that the least muscle and muscle fiber are activated to control the movement of joints according to the central nervous system ${ }^{13}$.

This study has a few limitations. All of the participants were right-handed and had had a right-sided hemiparetic stroke, but the lesion site was not included in the exclusion criteria. The sample size in this study was relatively small. Another potential limitation was the simplicity of the taping method used in the experiment. Other taping methods could perhaps facilitate upper extremity movements more efficiently. Further research on the relationship between various taping methods and upper extremity movements in stroke patients is necessary, particularly to investigate the specific lesion site.

In summary, taping facilitates the reaching movement of the taped arm of right-handed stroke patients but produces a negative effect on the untaped side. Previous studies, including brain imaging studies, support the positive effects of taping on the reaching movement of the taped arm. The negative effects on the reaching movement of the untaped arm could be due to the neuromotor associated with dual motor activity, taping, and reaching. The results of this study suggest that the relationship between taping and reaching movement should be considered in stroke rehabilitation. Further studies in other populations such as people with leftsided hemiplegia are needed in order to delineate the role of taping in upper extremity rehabilitation for stroke patients.

\section{REFERENCES}

1) Shinohara $T$, Usuda $S$ : Association of ability to rise from bed with improvement of functional limitation and activities of daily living in hemiplegic inpatients with stroke: a prospective cohort study. J Phys Ther Sci, 2010, 22: 29-34. [CrossRef]

2) Veerbeek JM, Kwakkel G, van Wegen EE, et al.: Early prediction of outcome of activities of daily living after stroke: a systematic review. Stroke, 2011, 42: 1482-1488. [Medline] [CrossRef]

3) Jaraczewska E, Long C: Kinesio taping in stroke: improving functional use of the upper extremity in hemiplegia. Top Stroke Rehabil, 2006, 13: 31-42. [Medline] [CrossRef]

4) Murphy PR, Hammond GR: Reversal of fusimotor reflex responses during locomotion in the decerebrate cat. Exp Physiol, 1997, 82: 837-858. [Medline] [CrossRef]

5) Lee MH, Lee CR, Park JS, et al.: Influence of kinesio taping on the motor neuron conduction velocity. J Phys Ther Sci, 2011, 23: 313-315. [CrossRef]

6) Kim KS, Seo HM, Lee HD: Effect of taping method on ADL, range of motion, hand function \& quality of life in post: stroke Patients for 5 weeks. Korean J Rehabil Nurs, 2002, 5: 7-17.

7) Maitra KK, Curry D, Gamble C, et al.: Using speech sounds to enhance occupational performance in young and older adults. OTJR (Thorofare, NJ), 2003, 23: 35-44.

8) Park JH, Yoo E, Chung B, et al.: Effects of vocalization on elbow motion during reaching in persons with hemiparetic stroke. NeuroRehabilitation, 2009, 25: 123-128. [Medline]

9) van Vliet PM, Sheridan MR: Ability to adjust reach extent in the hemiplegic arm. Physiotherapy, 2009, 95: 176-184. [Medline] [CrossRef]

10) Morris D, Jones D, Ryan H, et al.: The clinical effects of Kinesio ${ }^{\circledR}$ Tex taping: a systematic review. Physiother Theory Pract, 2013, 29: 259-270. [Medline] [CrossRef]

11) Grandi A, Mazzola M, Angelini L, et al.: Listening to the patient as a possible route to cost-effective rehabilitation: a case report. J Med Case Reports, 2012, 6: 19-22. [Medline] [CrossRef]

12) da Costa CS, Rodrigues FS, Leal FM, et al.: Pilot study: Investigating the effects of Kinesio Taping ${ }^{\circledR}$ on functional activities in children with cerebral palsy. Dev Neurorehabil, 2013, 16: 121-128. [Medline] [CrossRef]

13) Neumann DA: Kinesiology of the Musculoskeletal System: Foundation for Physical Rehabilitation. Milwaukee: Mosby, 2004. 